INPLASY

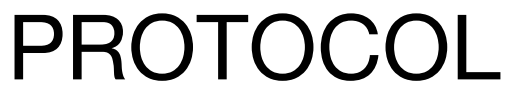

To cite: Lopes et al.

Comparison between the use of hydrogen peroxid and chlorhexidine solutions in intraoral antimicrobial control: systematic review. Inplasy protocol 202160004. doi: 10.37766/inplasy2021.6.0004

Received: 03 June 2021

Published: 03 June 2021

Corresponding author: Amanda Lopes

amandalopescontact@gmail.com

Author Affiliation:

Faculdade llapeo

Support: None.

Review Stage at time of this submission: Data extraction.

Conflicts of interest:

None declared.

\section{COMPARISON BETWEEN THE USE OF HYDROGEN PEROXID AND CHLORHEXIDINE SOLUTIONS IN INTRAORAL ANTIMICROBIAL CONTROL: SYSTEMATIC REVIEW}

Lopes, A¹; Miyasawa, E2; Sukekava, F³; Granjeiro, J4.

Review question / Objective: Pergunta pico em inglês: In adult patients who make use of solution for intraoral antimicrobial control, is there a difference in efficiency between hydrogen peroxide solutions and chlorhexidine solutions?

Condition being studied: Verify that hydrogen peroxide can be as efficient or better than chlorhexidine. Chemical plaque control is necessary for the maintenance of oral health, tissue repair processes and infection control.

Information sources: Databases: pubmed, COCHRANE library, web of science, scopus. Gray Literature: google scholar, open gray.

INPLASY registration number: This protocol was registered with the International Platform of Registered Systematic Review and Meta-Analysis Protocols (INPLASY) on 03 June 2021 and was last updated on 03 June 2021 (registration number INPLASY202160004).

\section{INTRODUCTION}

Review question / Objective: Pergunta pico em inglês: In adult patients who make use of solution for intraoral antimicrobial control, is there a difference in efficiency between hydrogen peroxide solutions and chlorhexidine solutions?
Condition being studied: Verify that hydrogen peroxide can be as efficient or better than chlorhexidine. Chemical plaque control is necessary for the maintenance of oral health, tissue repair processes and infection control. 


\section{METHODS}

Search strategy: The search was conducted on the foloowing data bases. pubmed, COCHRANE library, web of science, scopus, Google Academic, Grey Literature.

Participant or population: P (population/ patiente) - Adult.

Intervention: I (intervenction) - hydrogen peroxide.

Comparator: C (control) - chlorhexidine.

Study designs to be included: S (study) RCT.

Eligibility criteria: Inclusion criteria: RCT, mouthwash, adults, chlorhexidin, Hydrogen peroxide solutions / Exclusion criteria: Children, in vitro studies, in vivo study, gel, foam, toothpaste, concentrations above $2 \%$ clx and $1.5 \% \mathrm{H} 2 \mathrm{O} 2$, mixture between solutions, intra-channel washing, article unavailability for download.

Information sources: Databases: pubmed, COCHRANE library, web of science, scopus. Gray Literature: google scholar, open gray.

Main outcome(s): O (outcome) effectiveness in antimicrobial control.

Additional outcome(s): Plaque index; Gingival index; Bleending index; Probing depth.

Data management: The articles retrieved from the databases will be handled in RAYYAN; All data will be stored in an electronic spreadsheet; If meta-analysis is possible, Rev5 will be used.

Quality assessment / Risk of bias analysis: Rob2 - Cochrane Foundation.

Strategy of data synthesis: We will provide a narrative synthesis of the findings of the included studies, structured around the study design, number of participants, primary and secundary outcomes. We will provide summaries of the effects of the intervention for each study by calculating risk rates (for dichotomous results) or standardized mean differences (for continuous results). Only studies that present the same result with similar methodologies will be included in the paired meta-analysis. For continuous results (for example, GI, PI and GB), the estimated effects of the intervention can be expressed as mean difference (MD) with a 95\% confidence interval $(\mathrm{Cl})$. The inverse variance method can be used for random or fixed effect models.

\section{Subgroup analysis: None.}

Sensitivity analysis: Heterogeneity can be assessed using the chi-square, and the possible impact on the meta-analysis can be quantified via I-square. Values $=70 \%$ will be classified as medium and highly heterogeneous, respectively. When significant heterogeneity is found $(P<0.10)$, the results of the random effect model can be validated. When little heterogeneity is found, the fixed-effect model can be considered. The level of statistical significance can be determined as $P<0.05$. The data will be analyzed using the Review Manager statistical software (version 5.2.8; The Nordic Cochrane Center, The Cochrane Collaboration, Copenhagen, Denmark, 2014).

\section{Language: No language restrictions.}

\section{Country(ies) involved: Brazil.}

\section{Other relevant information: None.}

Keywords: chlorhexidine, hydrogen peroxide, oxygenating agents, mouthwashes, mouthrinses, rinses.

Dissemination plans: The resulted systematic review will be submitted to a periodical with selective process of pear review.

Contributions of each author:

Author 1 - Amanda Lopes - study design, data search, data collection, data extraction, manuscript elaboration. 
Email: amandalopescontact@gmail.com

Author 2 - Erton Miyasawa - study design, data search, data collection, data extraction, manuscript elaboration.

Email: ertonmassa@gmail.com

Author 3 - Flavia Sukekava - Expert analysis, data extraction, manuscript revision.

Author 4 - Jose Granjeiro - study design, data extraction, manuscript revision, final approval of the manuscript.

Email: jmgranjeiro@gmail.com 